\title{
Mean Field Dynamics in Non-Abelian Plasmas from Classical Transport Theory
}

\author{
Daniel F. Litim ${ }^{1, *}$ and Cristina Manuel ${ }^{2, \dagger}$ \\ ${ }^{1}$ Departament ECM, Facultat de Física \& IFAE, Universitat de Barcelona, Diagonal 647, E 08028 Barcelona, Spain \\ ${ }^{2}$ Theory Division, CERN, CH-1211 Geneva 23, Switzerland
}

(Received 1 March 1999)

\begin{abstract}
Based on classical transport theory, we present a general set of covariant equations describing the dynamics of mean fields and their statistical fluctuations in a non-Abelian plasma in or out of equilibrium. A procedure to obtain the collision integrals for the Boltzmann equation from the microscopic theory is described. As an application, we study a hot non-Abelian plasma close to equilibrium, where the fluctuations are integrated out explicitly. For soft fields, and at logarithmic accuracy, we obtain Bödeker's effective theory. [S0031-9007(99)09394-1]
\end{abstract}

PACS numbers: $12.38 . \mathrm{Mh}$

The dynamics of mean fields in non-Abelian plasmas is at the basis for an understanding of many properties of the early universe. This concerns the quark-gluon plasma which is believed to be formed at high temperature and/ or large chemical potential, or the plasma formed in the symmetric phase of the electroweak theory. The energy densities required to form a quark-gluon plasma might be reached in the coming generation of heavy ion colliders. A theoretical framework to describe the dynamics in non-Abelian plasmas in or out of equilibrium is thus mandatory (see [1] for an early review).

The dynamics of soft fields with momenta about $g^{2} T$ is dominated by nonperturbative phenomena, even for small gauge coupling $g \ll 1$ and close to equilibrium [2]. Here, some progress has been achieved recently by Bödeker [3], who derived an effective theory for the soft modes after integrating out the perturbative physical scales $T$ and $g T$ from the field theory.

In this Letter, we study mean field dynamics in the context of classical transport theory [4]. In the close-toequilibrium plasma, classical transport theory is known to describe correctly the physics around the scale $g T$ [5], in full agreement with the results obtained from quantum field theory [6]. We will show how also the physics at scales $g^{2} T$, and at logarithmic accuracy, can be understood in the same kinetic language. Our ultimate goal is providing a well-defined prescription to treat a number of interesting problems including out-of-equilibrium phenomena. Here, we present the main results of our analysis. This includes the derivation of a general set of dynamical equations for the mean fields and their fluctuations, and a prescription for obtaining collision integrals for Boltzmann equations. An explicit application for hot non-Abelian plasmas close to equilibrium is given as well. We leave for a longer publication a more extensive discussion and further details on the computations [7].

We begin with the derivation of a set of equations that describe both the dynamics of fluctuations and of the mean fields in a non-Abelian plasma. We follow the same philosophy and methods which have been used longly in kinetic theory to study Abelian plasmas (see [8]). Our equations can be seen as the generalization to non-Abelian plasmas.

The starting point is to consider gluons (or electrons, quarks) as charged point particles moving on a world line according to their classical equations of motion. They interact via a classical chromoelectromagnetic field. We consider a system of these particles carrying a nonAbelian color charge $Q^{a}$, where the color index runs from $a=1$ to $N^{2}-1$ for a $\mathrm{SU}(N)$ gauge group.

Within a microscopic description, the trajectories in phase space, and therefore the one-particle phase space density $f(x, p, Q)$, are known exactly. The classical trajectories $x(\tau), p(\tau)$, and $Q(\tau)$ are solutions of the Wong equations [9]:

$$
\begin{aligned}
& m \frac{d x^{\mu}}{d \tau}=p^{\mu}, \quad m \frac{d p^{\mu}}{d \tau}=g Q^{a} F_{a}^{\mu \nu} p_{\nu}, \\
& m \frac{d Q^{a}}{d \tau}=-g p_{\mu} f^{a b c} A_{b}^{\mu} Q_{c} .
\end{aligned}
$$

Here, $A_{\mu}^{a}$ denotes the microscopic vector gauge field, $F_{\mu \nu}^{a}[A]=\partial_{\mu} A_{\nu}^{a}-\partial_{\nu} A_{\mu}^{a}+g f_{a b c} A_{\mu}^{b} A_{\nu}^{c}$ is the corresponding microscopic field strength, and $f^{a b c}$ are the structure constants of $\mathrm{SU}(N)$. We set $c=\hbar=k_{B}=1$, and work in natural units. Effects of spin will be neglected.

The microscopic phase space density obeys Liouville's theorem $d f / d \tau=0$ [5]. We can write it using (1) as [4]

$$
p^{\mu}\left[\partial_{\mu}-g f^{a b c} A_{\mu}^{b} Q_{c} \partial_{a}^{Q}-g Q_{a} F_{\mu \nu}^{a} \partial_{p}^{\nu}\right] f=0,
$$

with $\partial_{\mu}^{p} \equiv \partial / \partial p^{\mu}$ and $\partial \stackrel{Q}{a} \equiv \partial / \partial Q^{a}$. This equation is gauge invariant, with $f$ transforming as a scalar [5], and is completed with the Yang-Mills equation,

$$
D_{\mu} F^{\mu \nu}(x)=J^{\nu}(x) \text {. }
$$

In the adjoint, the covariant derivative is given by $D_{\mu}^{a c}[A]=\partial_{\mu} \delta^{a c}+g f^{a b c} A_{\mu}^{b}$. In a self-consistent picture, the current $J_{a}^{\mu}(x)$ is due to the particles themselves, thus

$$
J_{a}^{\mu}(x)=g \sum_{\substack{\text { helicities } \\ \text { species }}} \int d P d Q p^{\mu} Q_{a} f(x, p, Q) .
$$


(Throughout, we shall omit a species or helicity index on $f$, and the sum over species and helicities will, in the sequel, not be given explicitly.) Physical constraints are enforced through the phase space volume element $d P \equiv$ $d^{4} p 2 \Theta\left(p_{0}\right) \delta\left(p^{2}-m^{2}\right) /(2 \pi)^{3}$, while $d Q$ contains $\delta$ functions imposing the group Casimirs (see [5] for their definition). The covariant conservation of the current (2c) is shown using (2a) [5].

If the system under study contains a large number of particles, it is impossible to follow their individual trajectories in phase space. Thus, $f$ can no longer be considered a deterministic quantity and one has to switch to a statistical description, taking statistical averages $\langle\cdots\rangle$ of all microscopic quantities. We write

$$
\begin{aligned}
& A_{\mu}^{a}=\bar{A}_{\mu}^{a}+a_{\mu}^{a}, \quad f=\bar{f}+\delta f, \\
& J_{a}^{\mu}=\bar{J}_{a}^{\mu}+\delta J_{a}^{\mu},
\end{aligned}
$$

where the quantities with a bar denote the mean values, e.g., $\bar{f}=\langle f\rangle$ and $\bar{A}=\langle A\rangle$, while the mean values of fluctuations vanish, $\langle\delta f\rangle=0$ and $\langle a\rangle=0$. We also split

$$
\begin{aligned}
F_{\mu \nu}^{a} & =\bar{F}_{\mu \nu}^{a}+f_{\mu \nu}^{a}, \\
f_{\mu \nu}^{a} & =\left(\bar{D}_{\mu} a_{\nu}-\bar{D}_{\nu} a_{\mu}\right)^{a}+g f^{a b c} a_{\mu}^{b} a_{\nu}^{c},
\end{aligned}
$$

with $\bar{D} \equiv D[\bar{A}]$ and $\bar{F} \equiv F[\bar{A}]$. Note that the mean field strength $\left\langle F_{\mu \nu}^{a}\right\rangle=\bar{F}_{\mu \nu}^{a}+g f^{a b c}\left\langle a_{\mu}^{b} a_{\nu}^{c}\right\rangle$ due to the nonAbelian nature of the theory.

Let us take a statistical average of (2) to find the kinetic equations for the mean values,

$$
\begin{aligned}
p^{\mu}\left(\bar{D}_{\mu}-g Q_{a} \bar{F}_{\mu \nu}^{a} \partial_{p}^{\nu}\right) \bar{f} & =\langle\eta\rangle+\langle\xi\rangle, \\
\bar{D}_{\mu} \bar{F}^{\mu \nu}+\left\langle J_{\text {fluc }}^{\nu}\right\rangle & =\bar{J}^{\nu} .
\end{aligned}
$$

In (5a) we used $\left[\partial_{\mu}-g f^{a b c} Q_{c} A_{\mu}^{b} \partial_{a}^{Q}\right] f \equiv D_{\mu} f$. The functions $\eta, \xi$, and $J_{\text {fluc }}$ are of second and higher order in the fluctuations and read

$$
\begin{aligned}
\eta & \equiv g Q_{a} p^{\mu} \partial_{p}^{\nu} f_{\mu \nu}^{a} \delta f, \\
\xi & \equiv g p^{\mu} f^{a b c} Q^{c}\left(\partial_{a}^{Q} a_{\mu}^{b} \delta f+g a_{\mu}^{a} a_{\nu}^{b} \partial_{p}^{\nu} \bar{f}\right), \\
J_{\text {fluc }}^{a, \nu} & \equiv g f^{d b c}\left(\bar{D}_{a d}^{\mu} a_{b, \mu} a_{c}^{\nu}+\delta_{a d} a_{b, \mu} f_{c}^{\mu \nu}\right) .
\end{aligned}
$$

The corresponding equations for the fluctuations are obtained by subtracting (5) from (2). The result is

$$
\begin{gathered}
p^{\mu}\left[\bar{D}_{\mu}-g Q_{a} \bar{F}_{\mu \nu}^{a} \partial_{p}^{\nu}\right] \delta f-g p^{\mu} a_{b, \mu} f^{a b c} Q_{c} \partial_{a}^{Q} \bar{f}-g Q_{a}\left[\bar{D}_{\mu} a_{\nu}-\bar{D}_{\nu} a_{\mu}\right]^{a} p^{\mu} \partial_{\nu}^{p} \bar{f}=\eta+\xi-\langle\eta+\xi\rangle, \\
{\left[\bar{D}^{2} a^{\mu}-\bar{D}^{\mu}\left(\bar{D}_{\nu} a^{\nu}\right)\right]^{a}+2 g f^{a b c} \bar{F}_{b}^{\mu \nu} a_{c, \nu}+J_{\text {fluc }}^{a, \mu}-\left\langle J_{\text {fluc }}^{a, \mu}\right\rangle=\delta J^{a, \mu} .}
\end{gathered}
$$

A number of comments are in order:

(i) Equations (5) and (7) are exact; no approximations have been made. In particular, they are also valid in outof-equilibrium situations.

(ii) Equations (5) and (7) are consistent with gauge invariance. They are covariant under the mean gauge field symmetry $\delta_{\epsilon} \bar{A}_{\mu}^{a}=\left(\bar{D}_{\mu} \epsilon\right)^{a}$ and $\delta_{\epsilon} a_{\mu}^{a}=g f^{a b c} a_{\mu}^{b} \epsilon^{c}$, in analogy to the background field formalism [10]. This establishes the compatibility of the statistical average with the gauge transformations of the mean field. We postpone a careful and detailed discussion to [7].

(iii) The microscopic current conservation implies

$$
\left(\bar{D}_{\mu} \bar{J}^{\mu}\right)_{a}+g f_{a b c}\left\langle a_{\mu}^{b} \delta J^{c, \mu}\right\rangle=0 .
$$

This is automatically consistent with (5b), provided $\bar{J}$ and $\delta J$ are solutions of (5) and (7). (A similar equation holds for the fluctuation fields.) Note that the validity of (8) turns into a nontrivial consistency check for approximate solutions.

(iv) The functions $\langle\eta\rangle$ and $\langle\xi\rangle$ can be considered as the effective collision integrals of the Boltzmann equation (5a). In our formalism, the collision integrals arise as correlators of statistical fluctuations. The fluctuations of the gauge fields cause random changes in the motion of particles, and, thus, they can be viewed as having the same effects as collisions. This can be seen as a derivation of collision integrals from the microscopic theory. Note also that the current induced by the fluctuations of the gauge field $\left\langle J_{\text {fluc }}\right\rangle$ is a purely non-Abelian effect.

(v) A general procedure for integrating out the fluctuations amounts to first solving their dynamics (7) in the background of mean fields. In general, this is a difficult task, in particular, due to the nonlinear terms in (7). The (explicit) solution is then inserted into (6). The back coupling of the fluctuations to the mean fields is finally obtained after taking the statistical average of the functions (6), and yields the effective collision integrals and the induced current in (5).

(vi) The set of equations (5) and (7) reproduces the known set of kinematic equations for Abelian plasmas in the corresponding limit [8], in which only the collision integral $\langle\eta\rangle$ survives. The Abelian counterpart of $\langle\eta\rangle$ can be expressed as the Balescu-Lenard collision integral [8]. One can then prove in a rigorous way the correspondence between fluctuations and collisions in the Abelian plasmas mentioned above. (An analogous derivation of collision integrals for Wigner functions can be found in [11]; see also [12].)

(vii) Neglecting all fluctuations reduces (5) to the wellknown (non-Abelian) Vlasov equations.

This terminates the derivation and discussion of the basic set of equations.

To put the method to work, we will specialize our analysis to hot non-Abelian plasmas close to equilibrium, with the gauge coupling $g \ll 1$. This allows us to perform several approximations. We will consider small fluctuations, neglecting in (6a) and (6c) the terms cubic in the fluctuations. This is interpreted as neglecting effective three body collisions versus binary ones. In the same spirit, we employ the second-moment approximation for the dynamics of the fluctuations [8], setting $\eta=\langle\eta\rangle, \xi=\langle\xi\rangle$, and $J_{\text {fluc }}=\left\langle J_{\text {fluc }}\right\rangle$ in (7). This linearizes the dynamics of the 
fluctuations and can be interpreted as neglecting the influence of collisions on the dynamics of the fluctuations. Finally, the term containing the mean field strength in (7a) is negligible compared to the remaining terms and will be omitted, as long as $g\left|\bar{F}_{a}^{\mu \nu}\right| / m_{D} \ll T$, where $m_{D}$ is the Debye mass [8].

We study the mean distribution function $\bar{f}(x, p, Q)=$ $\bar{f}^{\mathrm{eq}}\left(p_{0}\right)+g \bar{f}^{(1)}(x, p, Q)$. In the strictly classical approach, the relativistic Maxwell distribution at equilibrium is used for any species of particles. Here, we consider only massless particles in the adjoint, with $\int d Q Q_{a} Q_{b}=N \delta_{a b}$. For particles in the fundamental, one has $\int d Q Q_{a} Q_{b}=\frac{1}{2} \delta_{a b}$ instead. Solving (5) for vanset of non-Abelian hard thermal loops [5,6].

We now include small statistical fluctuations $\delta f$ around $\bar{f}$ and rewrite the approximations to (5) and (7) in terms of current densities and their fluctuations. Consider the current densities

$$
\begin{aligned}
& J_{a_{1} \cdots a_{n}}^{\rho}(x, p)=g p^{\rho} \int d Q Q_{a_{1}} \cdots Q_{a_{n}} f(x, p, Q), \\
& J_{a_{1} \cdots a_{n}}^{\rho}(x, v)=\int d \tilde{P} J_{a_{1} \cdots a_{n}}^{\rho}(x, p) .
\end{aligned}
$$
ishing fluctuations in this approximation gives the infinite

The measure $d \tilde{P}$ only integrates over the radial components, $d P=d \tilde{P} d \Omega / 4 \pi$, and $v^{\mu}=(1, \mathbf{v})$ with $\mathbf{v}^{2}=1$. The current $(2 \mathrm{c})$ is obtained performing the remaining angle integration $J(x)=\int \frac{d \Omega}{4 \pi} J(x, v)$. From now one, we will omit the arguments of the current density $J$, unless necessary to avoid confusion.

After multiplying (5a) by $g Q_{a} p^{\rho} / p_{0}$, summing over the two helicities, and integrating over $d \tilde{P} d Q$, we obtain for the mean current density at leading order in $g$

$$
\begin{aligned}
v^{\mu} \bar{D}_{\mu} \bar{J}^{\rho}+m_{D}^{2} v^{\rho} v^{\mu} \bar{F}_{\mu 0} & =\left\langle\eta^{\rho}\right\rangle+\left\langle\xi^{\rho}\right\rangle, \\
\bar{D}_{\mu} \bar{F}^{\mu \nu}+\left\langle J_{\text {fluc }}^{\nu}\right\rangle & =\bar{J}^{\nu},
\end{aligned}
$$

with the Debye mass $m_{D}^{2}=-2 g^{2} N \int d \tilde{P} d \bar{f}^{\mathrm{eq}}(p) / d p$, and

$$
\begin{aligned}
\eta_{a}^{\rho}=-g \int \frac{d \tilde{P}}{p_{0}}\{ & \left(\bar{D}_{\mu} a^{\rho}-\bar{D}^{\rho} a_{\mu}\right)^{b} \delta J_{a b}^{\mu}(x, p) \\
& \left.-\frac{p^{\rho}}{p_{0}}\left(\bar{D}_{\mu} a_{0}-\bar{D}_{0} a_{\mu}\right)^{b} \delta J_{a b}^{\mu}(x, p)\right\},
\end{aligned}
$$

$$
\xi_{a}^{\rho}=-g f_{a b c} v^{\mu} a_{\mu}^{b} \delta J^{c, \rho},
$$

$$
J_{\text {fluc }}^{\rho, a}=g f^{d b c}\left\{\bar{D}_{\mu}^{a d} a_{b}^{\mu} a_{c}^{\rho}+\delta^{a d} a_{\mu}^{b}\left(\bar{D}^{\mu} a^{\rho}-\bar{D}^{\rho} a^{\mu}\right)^{c}\right\} .
$$

For the fluctuations, we find

$$
\left[\bar{D}^{2} a^{\mu}-\bar{D}^{\mu}(\bar{D} a)\right]_{a}+2 g f_{a b c} \bar{F}_{b}^{\mu \nu} a_{c, \nu}=\delta J_{a}^{\mu} .
$$

We solve the equations for the fluctuations (12) with an initial boundary condition for $\delta f$, and $a_{\mu}(t=0)=0$. Exact solutions to (12a) and (12b) can be obtained [7]. The current fluctuation $\delta J_{a}$ reads, for $x_{0} \equiv t \geq 0$,

$$
\begin{aligned}
\delta J_{a}^{\rho}(x, v)= & \bar{U}_{a b}\left(x, x_{t}\right) \delta J_{b}^{\rho}\left(x_{t}, v\right)-\int_{0}^{\infty} d \tau \bar{U}_{a b}\left(x, x_{\tau}\right) \\
& \times\left\{g f_{b d c} v^{\mu} a_{\mu}^{d}\left(x_{\tau}\right) \bar{J}_{c}^{\rho}\left(x_{\tau}, v\right)\right. \\
& \left.\quad+m_{D}^{2} v^{\rho} v^{\mu}\left(\bar{D}_{\mu} a_{0}-\bar{D}_{0} a_{\mu}\right)^{b}\left(x_{\tau}\right)\right\} .
\end{aligned}
$$

We introduced $x_{\tau} \equiv x-v \tau$ and the parallel transporter $\bar{U}_{a b}$, obeying $\left.v^{\mu} \bar{D}_{\mu}^{x} \bar{U}_{a b}(x, y)\right|_{y=x_{\tau}}=0$. In order to solve (12c) for $a_{\mu}$, we make a double expansion in both $\bar{A}$ and $\bar{J}$, using $\bar{U}_{a b}=\delta_{a b}+\mathcal{O}(g \bar{A})$. We denote by $a^{(n)}$ the term containing a total of $n$ powers in the mean fields $\bar{A}$ and/or $\bar{J}$.

For our purposes, it will be sufficient to consider the zeroth order term in $\bar{A}$, and the zeroth and first order terms in $\bar{J}$. Using the one-sided Fourier transform [8], we find $a_{i, a+}^{T(0)}(k)=\frac{1}{-k^{2}+\Pi_{T}} \int \frac{d \Omega_{\mathbf{v}}}{4 \pi} \frac{\delta J_{i, a}^{T}(t=0, \mathbf{k}, v)}{-i k \cdot v}$,

$$
\begin{aligned}
a_{i, a+}^{T(1)}(k)= & \frac{-g f_{a b c}}{-k^{2}+\Pi_{T}} P_{i j}^{T}(\mathbf{k}) \int \frac{d \Omega_{\mathbf{v}}}{4 \pi} \frac{1}{-i k \cdot v} \\
& \times \int \frac{d^{4} q}{(2 \pi)^{4}} v^{\mu} a_{\mu}^{b(0)}(q) \bar{J}_{j}^{c}(k-q, v),
\end{aligned}
$$

in the gauge $\mathbf{k} \cdot \mathbf{a}=0$. The function $\Pi_{T}(k)$ is the transverse polarization tensor of the plasma, $P_{i j}^{T}(\mathbf{k})=$ $\delta_{i j}-k_{i} k_{j} / \mathbf{k}^{2}$ is the transverse projector, and $a_{i}^{T} \equiv$ $P_{i j}^{T} a_{j}$. Retarded boundary conditions are assumed above, with the prescription $k_{0} \rightarrow k_{0}+i 0^{+}$.

With the above, we can express all fluctuations in terms of initial conditions and the mean fields. Following [8] the statistical average over initial conditions can be deduced [7] and finally expressed (for each species or helicity index) as

$$
\begin{aligned}
\left\langle\delta f_{\mathbf{k} p Q} \delta f_{\mathbf{k}^{\prime} p^{\prime} Q^{\prime}}\right\rangle= & (2 \pi)^{6} \delta^{(3)}\left(\mathbf{k}+\mathbf{k}^{\prime}\right)\left[\delta^{(3)}\left(\mathbf{p}-\mathbf{p}^{\prime}\right)\right. \\
& \left.\times \delta\left(Q-Q^{\prime}\right) \bar{f}(p)+\mu_{\mathbf{k} p p^{\prime} Q Q^{\prime}}\right]
\end{aligned}
$$

The function $\delta\left(Q-Q^{\prime}\right)$ is, apart from a (representation dependent) normalization constant, a $N^{2}-N$ dimensional $\delta$ function over the proper set of Darboux variables related to the color charges [5]. The second term in (15) is the Fourier transform of a smooth function that vanishes at large distances. The above statistical average is all we need to evaluate the collision integrals.

For the remaining part, we will concentrate on the dynamics of mean fields with typical momenta around $g m_{D}$. When computing the related collision integrals, we will find logarithmic divergences, cut off in the infrared 
by the inverse collision time. We employ the leading logarithmic approximation, assuming $\ln (1 / g) \gg 1$ while neglecting all subleading (though finite) terms.

We find that the induced current $\left\langle J_{\text {fluc }}^{(0)}\right\rangle$ vanishes, as do the fluctuation integrals $\left\langle\eta^{(0)}\right\rangle$ and $\left\langle\xi^{(0)}\right\rangle$. The vanishing of $\left\langle\eta^{(0)}\right\rangle$ is consistent with the fact that in the Abelian limit the counterpart of $\langle\eta\rangle$ vanishes at equilibrium [8]. In the same spirit, we evaluate the collision integrals containing one $\bar{J}$ field. Consider

$$
\begin{aligned}
\left\langle\xi_{\rho, a}^{(1)}\right\rangle= & g f_{a b c} v^{\mu}\left\{-\left\langle a_{\mu, b}^{(1)}(x) \delta J_{\rho, c}^{(0)}(x, v)\right\rangle+g f_{c d e} v^{\nu}\right. \\
& \left.\times \int_{0}^{\infty} d \tau \bar{J}_{\rho, e}\left(x_{\tau}, v\right)\left\langle a_{\mu, b}^{(0)}(x) a_{\nu, d}^{(0)}\left(x_{\tau}\right)\right\rangle\right\},
\end{aligned}
$$

which simplifies, at logarithmic accuracy, to

$$
\begin{aligned}
&\left\langle\xi_{\rho, a}^{(1)}(x, v)\right\rangle=-\frac{g^{2}}{4 \pi} N T \ln (1 / g) \\
& \times v_{\rho} \int \frac{d \Omega_{\mathbf{v}^{\prime}}}{4 \pi} I\left(\mathbf{v}, \mathbf{v}^{\prime}\right) \bar{J}_{a}^{0}\left(x, v^{\prime}\right), \\
& I\left(\mathbf{v}, \mathbf{v}^{\prime}\right) \equiv \delta^{(2)}\left(\mathbf{v}-\mathbf{v}^{\prime}\right)-\frac{4}{\pi} \frac{\left(\mathbf{v} \cdot \mathbf{v}^{\prime}\right)^{2}}{\sqrt{1-\left(\mathbf{v} \cdot \mathbf{v}^{\prime}\right)^{2}}} .
\end{aligned}
$$

The above expression has been obtained first in [3], and reproduces the collision integral considered in the Boltzmann equation of [13].

We verified that the leading logarithmic solution is consistent with gauge invariance. Evaluating the correlator in (8) yields $\bar{D}_{\mu} \bar{J}^{\mu}=0$, in accordance with (10b) in the present approximation.

Following Bödeker, one can now estimate $\bar{J}^{i}$ from (10a) to obtain for (10b)

$$
\left(\bar{D}_{\mu} \bar{F}^{\mu i}\right)_{a}=\sigma \bar{E}_{a}^{i}+\nu_{a}^{i}, \quad \sigma=\frac{4 \pi m_{D}^{2}}{3 N g^{2} T \ln (1 / g)} .
$$

This is the result of [3]. The coefficient $\sigma$ represents the color conductivity and has been discussed in $[13,14]$. The white noise $\nu$ has its origin in the fluctuations of the transverse part of $\xi^{(0)}[3,7]$. We obtain to leading order

$$
\left\langle\nu_{a}^{i}(x) \nu_{b}^{j}(y)\right\rangle=2 T \sigma \delta^{i j} \delta_{a b} \delta^{(4)}(x-y),
$$

in accordance with the fluctuation-dissipation theorem (FDT). Note also that the classical Debye mass differs from the quantum one.

In order to go beyond classical transport theory, we expand about the bosonic (fermionic) quantum-statistical equilibrium distribution function $\bar{f}_{+}\left(\bar{f}_{-}\right)$. For gluons in the adjoint, the Debye mass obtains as $m_{D}^{2}=g^{2} N T^{2} / 3$. The FDT is obeyed as well, if $\bar{f}$ in (15) is replaced by $\bar{f}_{ \pm}\left(1 \pm \bar{f}_{ \pm}\right)$. (This should, however, be derived in a similar way as (15) from the microscopic theory [7].) Also, the quantum collision integrals are obtained with the correct statistical factors [7]. It is interesting to note that all quantum modifications are contained in the implicit change of $m_{D}$.
This terminates the explicit derivation, in the leading logarithmic approximation, of the collision integral and the dynamical equations for the soft fields from classical transport theory.

In summary, we have given a prescription to derive mean gauge field equations from classical transport theory. This includes a recipe to obtain effective (classical or quantum) collision integrals from the microscopic theory. The approach is in accordance with gauge invariance. In a close-to-equilibrium plasma and for small gauge coupling, we reproduce Bödeker's effective theory.

The last part of our analysis can straightforwardly be generalized in order to obtain explicit expressions for the collision integrals not only for the soft momentum region. Another interesting open problem is using the same methods for out-of-equilibrium situations. Based on the evaluation of collision integrals for Abelian plasmas out of equilibrium [8], we should find the Coulomb logarithm changing drastically the mean non-Abelian gauge field equations.

It remains remarkable that classical transport theory is efficient enough as to describe not only the non-Abelian dynamics of semihard modes with momenta around $g T$, but as well the nonperturbative dynamics of soft gluons at leading logarithmic order. This establishes a link even beyond the one-loop level between our approach and a complete quantum field theoretical treatment, whose deeper structure is waiting to be uncovered [15].

*Email address: litim@ecm.ub.es

${ }^{\dagger}$ Email address: cristina.manuel@cern.ch

[1] H.-Th. Elze and U. Heinz, Phys. Rep. 189, 81 (1989).

[2] D. J. Gross, R. D. Pisarski, and L.G. Yaffe, Rev. Mod. Phys. 53, 43 (1981).

[3] D. Bödeker, Phys. Lett. B 426, 351 (1998).

[4] U. Heinz, Phys. Rev. Lett. 51, 351 (1983); Ann. Phys. (N.Y.) 161, 48 (1985); 168, 148 (1986).

[5] P. R. Kelly, Q. Liu, C. Lucchesi, and C. Manuel, Phys. Rev. Lett. 72, 3461 (1994); Phys. Rev. D 50, 4209 (1994).

[6] R. D. Pisarski, Phys. Rev. Lett. 63, 1129 (1989); E. Braaten and R.D. Pisarski, Nucl. Phys. B337, 569 (1990); J. P. Blaizot and E. Iancu, Phys. Rev. Lett. 70, 3376 (1993); Nucl. Phys. B417, 608 (1994).

[7] D. F. Litim and C. Manuel (to be published).

[8] Yu. L. Klimontovich, Statistical Physics (Harwood Academic, Chur, Switzerland, 1986); E. Lifshitz and L. Pitaevskii, Physical Kinetics (Pergamon, Oxford, 1981).

[9] S. Wong, Nuovo Cimento A 65, 689 (1970).

[10] L. F. Abbott, Nucl. Phys. B185, 189 (1981); H.-Th. Elze, Z. Phys. C 47, 647 (1990).

[11] A. V. Selikhov, Phys. Lett. B 268, 263 (1991); Phys. Lett. B 285, 398(E) (1992).

[12] A. V. Selikhov and M. Gyulassy, Phys. Rev. C 49, 1726 (1994).

[13] P. Arnold, D. T. Son, and L. G. Yaffe, hep-ph/9810216.

[14] A. V. Selikhov and M. Gyulassy, Phys. Lett. B 316, 373 (1993); H. Heiselberg, Phys. Rev. Lett. 72, 3013 (1994).

[15] R. D. Pisarski, hep-ph/9710370. 